\title{
From the Perspective of Marx's Labor Theory of Value on the Value Creation of China's Cultural Industry
}

\author{
Shan Dongfang ${ }^{1, a}$ \\ ${ }^{1}$ School of economics, Shanxi University of Finance and Economics, Taiyuan, Shanxi, China \\ a605760023@qq.com
}

\begin{abstract}
Based on the perspective of Marx's labor value theory, this paper analyzes whether the labor in the cultural industry can create value, and comes to the conclusion that not the whole labor in the cultural industry can create value. At the same time, this paper deeply analyzes the current situation of China's cultural industry in value creation from the two aspects of self value creation and social value creation. On this basis, taking improving the value creation ability of living labor as the core, this paper constructs VAR model from the four aspects of scientific research investment, fixed asset investment, patent authorization and economic policy uncertainty, This paper studies its impact on the value creation ability of cultural industry. Although production factors such as science and technology, capital and human capital investment cannot create value, they have the ability to affect the value creation of the whole cultural industry.
\end{abstract}

Keywords: culture, cultural industry, labor value theory, value creation.

\section{马克思劳动价值论视角下我国文化产业价值创造探究}

单东方 $1, \mathrm{a}$

\author{
${ }^{1}$ 山西财经大学经济学院, 太原, 山西, 中国 \\ a605760023@qq.com
}

\section{摘要}

基于马克思劳动价值论的视角，对文化产业劳动是否创造价值做了分析，得出了并非整个文化产业的劳动都能 创造价值的结论。同时从自身价值创造与社会价值创造两方面深入分析了我国文化产业在价值创造上的现状， 并以此为基础，以提升活劳动价值创造能力为核心，从科研投入、固定资产投资、专利授权、经济政策不确定 性四方面，通过构建 VAR 模型，研究了其对文化产业价值创造能力的影响。虽然科学技术、资金、人力资本投 资等生产要素不能创造价值，但具有影响整个文化产业价值创造的能力。

关键词: 文化，文化产业，劳动价值论，价值创造

\section{1. 引言}

随着全球经济逐步迈入后工业化时代, 人们不再 满足于以往简单的温饱需求, 在需求层面上更加注重 精神方面的提高。文化作为一个民族最基本的象征, 从文化中应运而生的文化产业, 是生产力发展到一定 阶段的必然产物, 符合经济形态演变的客观经济规 律。一个国家, 当文化表现出比物质和货币资本更强 大力量的时候, 也表示着国家具备了进入更高的发展 阶段的潜力, 未来才能更好为社会发展助力。 ${ }^{[1]}$ 然而
在马克思劳动价值论视角下的文化产业劳动是否属 于生产劳动, 哪些非物质生产领域的劳动创造价值, 以及我国文化产业发展现阶段下文化产业价值创造 受何种因素影响更大等问题仍需要进行进一步的探 讨, 这有助于文化产业的可持续发展。

\section{2. 文献综述}

关于价值创造的研究主要分为两个方面, 一个是 对于什么劳动创造价值, 非物质生产部门的劳动是否 
创造价值的理论分析上, 一个是对产业或企业价值创 造效果及能力衡量测定上的实证分析。

由于马克思所处资本主义工业化时期, 以机器大 工业生产为背景, 在生产力水平低下的当时, 商品主 要表现为简单体力劳动下的物质产品, 因而也就更重 视物质产品的生产性劳动。然而随着人类逐步步入信 息化、知识化时代, 生产、生活方式、思维方式发生 了革命性变革, 生产性劳动大幅度削减, 劳动创造价 值的形式、作用方式、作用内容越来越丰富, 所以众 多中外学者开始对马克思的 “劳动一元论” 展开探讨。 比较主流的观点大多认为要以当前实际对马克思的 劳动价值论进行一定内涵上的拓展。如萧灼基 (2001) 指出马克思意义上的生产劳动, 主要指 “直接生产过 程的劳动”, 同时也包含 “直接生产过程在流通领域 继续的劳动” , 即运输、包装、保管的劳动。 ${ }^{[2]}$ 李玲 娥 (2002) 认为生产劳动等的概念不是一成不变的, 应从注重研究创造价值劳动的外延, 转向注重研究其 内涵。 ${ }^{[3]}$ 产业或企业价值创造效果及能力衡量测定上 的实证分析上, 董淑兰等 (2014) 发现, 盈利能力的 强弱影响产业价值创造能力的提高, 但就企业规模而 言, 在一定程度上会抑制价值创造能力水平。 ${ }^{[4]}$ 就文 化产业价值创造而言, 仍停留在理论分析的层面, 李 庭新, 李书 (2005) 将文化产品的价值与一般商品的 价值做了区分, 并构建了文化产品价值的模型, 模型 中不仅包含隐含在产品内的知识价值、文化产品价值 增值的年利率、文化产品在使用过程中价值的转移, 同时将文化生产者在价值创造时所增加的文化价值 一并加以概括, 但鉴于数据不好衡量, 各个文化产品 差异大, 文章并未给出实证分析。 ${ }^{[5]}$

综上所述, 目前对文化产业价值创造的相关研究 较少, 而且大多着重与理论上的探讨, 在文化产业快 速发展的今天有必要以马克思劳动价值论为核心对 我国文化产业价值创造能力的现状及影响因素等进 行相关实证分析与检验, 为提升我国文化产业价值创 造能力提出建议。

\section{3. 劳动价值论下文化产业价值创造分析}

\section{1 文化产业价值创造的理论分析}

马克思分析劳动价值论的开始是商品, 所以在对 文化产业的劳动是否创造价值的讨论也从文化商品 开始, 鉴于本文对以实物形态和服务形态向文化消费 者提供的商品或服务统称为文化产品, 而无论是提供 有形的诸如图书、杂志等实物形态的物质产品, 还是 提供表演等文化服务的演员等, 我们都不能否认他的 有用性, 其所提供的服务都直接或间接的服务于人们 的生产、生活, 都是用来交换的劳动产品, 因而都具 有商品的二因素。从价值上看, 文化产品的的价值是 凝结在文化产品中的人类一般劳动; 从使用价值上 看, 文化产品的使用价值是文化对于人而言带来的精 神层面上的效益或者有用性。
其次，提供文化产品的劳动是否创造价值。马克 思过去将在物质生产领域的劳动定义为生产性劳动, 生产性劳动创造价值。 ${ }^{[6]}$ 但现今的生产性劳动概念已 有了新的拓展, 伴随经济结构剧烈的变革, 产业结构 的不断优化升级, 非物质生产部门的服务劳动也逐渐 被纳入到创造价值的劳动的范畴里。生产以实物形态 呈现的文化产品的劳动, 即为直接满足人们的精神需 要而进行的创作、制造、传播、展示等文化产品的直 接或间接的生产活动必然是创造价值的，同时鉴于物 流、仓储等为生产领域服务的非生产性劳动也创造价 值, 那么根据文化产业的涵盖范围, 相关劳动也是创 造价值的。还有一部分即提供表演等文化服务的演员 等文化工作者的劳动是否创造价值。本文认为是创造 价值的，因为此类文化工作者提供的服务同样是文化 产品生产过程中的劳动, 是属于生产性劳动的, 只不 过产品并非以实物的形态表现出来而已, 但为文化产 品的推广所进行的销售等劳动则不能创造价值, 从此 也可看出并非整个文化产业的劳动都能创造价值。

最后, 马克思在研究价值创造时的主体即为商 品, 那么基于劳动价值论对文化产业价值创造进行分 析实质上也就是对文化产品价值创造进行分析。然而 这种抽象劳动下创造的价值是难以衡量的, 文化产品 的价值创造所用的社会必要劳动时间也无法简单的 获得。因而本文在对文化产业价值创造的分析上，不 局限于文化产品自身的价值创造, 将更多价值创造表 现加以衡量。

\section{2 我国文化产业价值创造现状分析}

由于价值创造的过程就是追求实现包括自身价 值和社会价值在内的价值最大化。因而我国文化产业 价值创造的相关研究也从这两个方面展开。

在自身价值创造上，鉴于文化产品的特殊性，其 除了物质价值之外, 更注重精神层面的价值, 然而这 种相对较为隐形的价值并不好衡量, 本文从文化产业 在经济上的表现来分析。总体来说，我国文化及相关 产业已经成为了当前经济增长的一个新的驱动力, 文 化产品出口额持续上升, 文化产品贸易连续实现顺 差。在总量持续稳步增长的同时, 占 GDP 的比重也逐 渐上升, 但整体而言我国与世界主要国家相比仍与其 存在一定差距, 且并不是每种文化产品都能获得良好 的收益, 虽然文化产品整体在出口创汇上表现良好, 但在许多具体产品的出口上仍具有较大发展潜力。同 时, 随着我国进入新常态以来, 文化产业的需求收入 弹性是大于 1 的, 可见随着以 “互联网+” 为引领的 新消费模式的带动, 文化产业无疑有着巨大的价值创 造潜力。

在社会价值创造上，文化产业作为横跨第二产业 和第三产业两大产业的多元产业, 其自身的发展一方 面促进文化、娱乐和旅游休闲为代表的服务业就业的 增加, 另一方面也使得辅助文化娱乐活动的设备制造 
的轻工业产值增加, 从而拉动就业增加。1近十年来 文化产业从业人员数呈波动上涨的趋势, 其中女性从 业人员占比近一半, 但文化产业从业人员占全部从业 人员的比重仍然较低。社会效益上, 由于产业的社会 效益具有滞后性, 非显性的特点, 以及数据的可得性 偏低，很难对其划分定量的标准。

总体来说, 我国文化产业价值创造的现状相对较 好, 但国际竞争力、就业等方面仍存在提升的空间, 伴随产业规模的不断加大, 出口的提升, 高附加值的 文化产品在高需求收入弹性下有非常大的需求空间, 价值创造能力有望得到进一步的提升。

\section{4. 文化产业价值创造能力影响因素分析}

\section{1 数据来源及模型假定}

文化产业价值创造的表现是多方面, 为了更好提 升文化产业价值创造的能力, 同时鉴于数据的可获得 性, 本文采用年度数据, 选取文化及相关产业增加值 CIVA 为被解释变量, 以其代表文化产业价值创造能 力的高低。同时以提高活劳动创造价值能力为核心, 选取文化产业固定资产投资 I、科技投入 R\&D、专利 授权 GPA、产业政策不确定性 EPU 四项指标, 分别考 察其对 CIVA 是否有影响。相关数据整理自历年中国 文化及相关产业统计年鉴。

本文采取 $\mathrm{P}$ 阶向量自回归模型: $Y_{t}=A_{1} Y_{t}+A_{2} Y_{t-2}+\ldots+A_{p} Y_{t}-p+\varepsilon_{\mathrm{t}}$

其中, Yt 代表 CIVA, 内生变量有 P 阶滞后期, $\varepsilon \mathrm{t}$ 为随机扰动项。

\section{2 实证分析}

\section{2.1 单位根检验}

为消除异方差的影响, 对相关数据的时间序列数 据分别进行取对数的相关处理, 用 LCIVA、LI、LRD、 LGPA、LEPU 分别表示其取对数后的值。为保证 VAR 模型的有效性, 首先对时间序列数据进行单位根检 验, 本文采用 ADF (Augmented Dickey Fuller) 方 法对数据的平稳性做单位根检验, 检验结果在 $10 \%$ 的 显著性水平下, 5 变量的二阶差分是平稳的。

\subsubsection{VAR 稳定性检验}

采用 AR 特征多项式对 VAR 模型进行稳定性检验, AR 特征多项式的特征根都在单位圆内, 表明 VAR 模 型是平稳的。

\subsection{3 脉冲响应函数}

脉冲响应函数即在扰动项上加一个标准差大小
的冲击, 对于内生变量当前值和未来值所带来的影 响, 如图 1 所示。
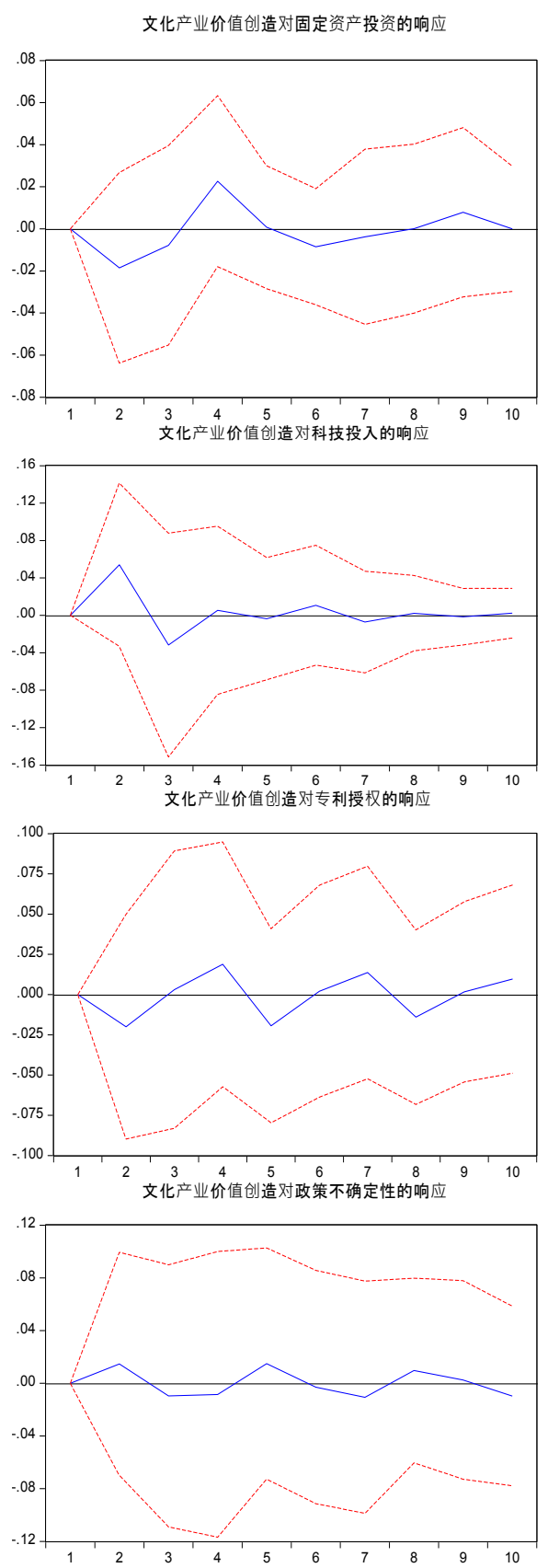

图 1 脉冲响应函数图

鉴于样本量较小，并没有得到最好的脉冲效果， 但从部分图中仍可看出明显的收敛趋势。图 1-1 表示 的是文化产业价值创造对固定资产投资的响应，可见 在初期对价值创造施加一个正向冲击后，反而会对其 增长率产生负向的抑制作用, 在第二期达到最低值, 三期后转为正向反馈并逐步向 0 收敛。这种响应趋势 可以从现实意义上加以理解，固定资产投资的增加并 不能同时促进文化产业价值创造能力的提升, 对其的 影响是有滞后效应的, 基于成本考量、风险控制等种 种因素可能会造成对其的抑制作用, 但这种抑制作用 会随时间而逐步减弱。图 1-2 是文化产业价值创造对 科技投入的反馈，可以看出其反馈作用十分明显。给 
定其一个正向冲击, 文化产业价值创造效果显著, 效 应系数为 0.04 , 在第四期后逐步向 0 收玫。从中可 见科研投入对提升文化产业价值的重要作用, 这在一 定程度上也反映了文化产品的特殊性, 知识产权、文 化创意在技术的支撑下可以快速体现在价值创造上。 图 1-3、1-4 分别是专利授权和政策不确定性对文化 产业价值创造的影响。脉冲效果并不明显, 一方面可 能是由于样本量小造成的, 另一方面也可能是我国文 化产品专利授权在直接转化为价值增值上的作用还 不明显, 同时政策的不确定性对文化产业价值创造的 影响还不大, 不能称其为影响因素。

\section{5. 结论}

在社会经济飞速发展，人类生产、生活方式发生 革命性变革的今天, 马克思劳动价值论也要与时俱进 的继承和发展。对于价值创造, 在拓展生产性劳动的 基础上, 本文认为生产以实物形态呈现的文化产品的 劳动, 提供表演等文化服务的演员等文化工作者的劳 动都是创造价值的, 但为文化产品的推广所进行的销 售等劳动则不能创造价值, 即并非整个文化产业的劳 动都能创造价值。

除了对文化产业相关劳动是否创造价值的理论 分析, 本文从自身价值创造与社会价值创造深入分析 了我国文化产业在价值创造上的现状, 并以此为基 础, 以提升活劳动价值创造能力为核心, 从科研投入、 固定资产投资、专利授权、经济政策不确定性四方面, 通过构建 VAR 模型, 分别研究了其对文化产业价值创 造能力的影响。虽然科学技术、资金、人力资本投资 等生产要素不能创造价值, 但具有影响整个文化产业 价值创造的能力, 尤其是科学技术的提升可以有效促 进劳动生产率的提高, 进而促进整个文化产业的发
展。但同时也要注意我国文化产业价值创造对经济政 策不确定性的反馈作用不明显, 这从一定层面上体现 出, 虽然政府在制定政策时要尽可能保持宏观经济政 策的持续性、稳定性，但就文化产业而言，可以采用 更为灵活的调整战略, 通过对提升价值创造能力影响 因素的其他方面予以积极的政策支持, 从而提升文化 产业价值创造的能力。

\section{REFERENCES}

[1] Jiao Binlong. (2014) Economics of cultural industry. Higher education press. TaiYuan. pp.71-72

[2] Xiao Zhuoji. (2001) Promoting theoretical innovation and guiding economic practice -- on "deepening the understanding of labor and labor value theory". contemporary economic research. J. 04-05

[3] Li linge. (2002) 4A review of socialist labor and labor value theory. Journal of Shanxi University of Finance and economics.J.10:03

[4] Dong Shulan, Wang Xiaowei etc. (2014) Research on the influencing factors of enterprise value creation ability -- empirical data from Heilongjiang Province. Journal of Heilongjiang Bayi Agricultural Reclamation University. J.04:137

[5] Li Tingxin, Li Shu. (2005) Economic analysis of the value of cultural products. Market Weekly. 05: 93-94

[6] Marx Surplus value theory. (1975) People's publishing house Volume I. pp.436 